A Scientific/Technical Report for the

Advanced Detector Research Program

\title{
Large-Scale Testing and Development of Gadolinium Trichloride for Use in Neutron Detection in Large Water Cherenkov Detectors
}

Closeout Award DE-FG02-05ER41386

October 1, 2005 - September 30, 2006

\author{
Principal Investigator: \\ Mark R. Vagins \\ Research Physicist \\ University of California, Irvine
}

Prepared for the Division of High Energy Physics of the Office of Science (SC), U.S. Department of Energy 


\section{Contents}

1 Physics of Neutron Capture 2

1.1 In Pure Water . . . . . . . . . . . . . . . . . . . 2

1.2 In the Presence of a Solute . . . . . . . . . . . . . 2

2 Why Gadolinium Trichloride? 3

2.1 Solubility ................... . . . 3

2.2 High Efficiency for Producing Observable $\gamma$-rays . . . . . . . . 4

3 Physics Reach of WC Detectors with $\mathbf{G d C l}_{3}$

3.1 Relic Supernova Neutrinos . . . . . . . . . . . . . . . 5

3.2 Galactic Supernova Neutrinos . . . . . . . . . . . . . 6

3.3 Reactor Anti-neutrinos . . . . . . . . . . . . . . 6

3.4 Atmospheric Neutrinos . . . . . . . . . . . . . . . . 7

3.5 Long Baseline Neutrinos . . . . . . . . . . . . . . . 8

3.6 Proton Decay . . . . . . . . . . . . . . . . . 9

4 Program of Study $\quad 9$

4.1 The One Kiloton Detector at KEK . . . . . . . . . . . . . . 10

5 Results of the Study $\quad 12$

5.1 Water Transparency in the $1 \mathrm{KT}$. . . . . . . . . . . . . 12

5.2 Water Purification System of the $1 \mathrm{KT}$. . . . . . . . . . . 14

5.3 Conclusions .................... 16 


\begin{abstract}
Water Cherenkov detectors have been used for many years as inexpensive, effective detectors for neutrino interactions and nucleon decay searches. While many important measurements have been made with these detectors a major drawback has been their inability to detect the absorption of thermal neutrons.

We believe an inexpensive, effective technique could be developed to overcome this situation via the addition to water of a solute with a large neutron cross section and energetic gamma daughters which would make neutrons detectable. Gadolinium seems an excellent candidate especially since in recent years it has become very inexpensive, now less than $\$ 8$ per kilogram in the form of commercially-available gadolinium trichloride, $\mathrm{GdCl}_{3}$. This non-toxic, non-reactive substance is highly soluble in water. Neutron capture on gadolinium yields a gamma cascade which would be easily seen in detectors like Super-Kamiokande.

We have begun to investigate the use of $\mathrm{GdCl}_{3}$ as a possible upgrade for the Super-Kamiokande detector with a view toward improving its performance as a detector for atmospheric neutrinos, supernova neutrinos, wrong-sign solar neutrinos, reactor neutrinos, proton decay, and also as a target for the coming T2K long-baseline neutrino experiment. This large-scale investigation, conducted in the one kiloton water Cherenkov detector built for the $\mathrm{K} 2 \mathrm{~K}$ long-baseline experiment, follows up on highly promising benchtop-scale work previously carried out with the assistance of a 2003 Advanced Detector Research Program grant.
\end{abstract}



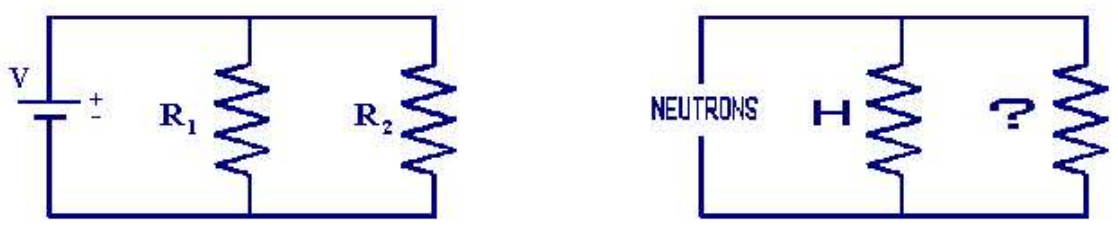

Figure 1: Resistors in parallel compete for electric current (left) just as multiple neutron-capturing elements in solution compete for neutrons (right).

\section{Physics of Neutron Capture}

\subsection{In Pure Water}

Neutrons liberated in water by the inverse beta reaction $\bar{\nu}_{e}+p \rightarrow e^{+}+n$ (and other processes) are quickly thermalized. On average it takes about twenty collisions with the water's free protons over the course of $\sim 10 \mu$ s to bring a neutron emitted with a few $\mathrm{MeV}$ down to room temperature $(0.025 \mathrm{eV})$.

Once thermalized, and after bouncing around for another $100 \mu \mathrm{s}$ or so, the neutron is captured by a proton or oxygen nucleus in the water. The cross sections for these capture reactions are 0.33 barns and 0.19 millibarns, respectively, so to first approximation every thermal neutron is captured on a free proton via the reaction $n+p \rightarrow d+\gamma$.

The resulting gamma has an energy of $2.22 \mathrm{MeV}$ and makes very little detectable light since the Compton scattered electron is close to Cherenkov threshold. Hence, in traditional water Cherenkov detectors (which tend to have trigger thresholds around $5 \mathrm{MeV}$ ) these neutron captures are generally not recorded and consequently there is no way to distinguish between neutrino interactions and anti-neutrino interactions on free protons.

\subsection{In the Presence of a Solute}

However, the situation can be quite different if the right substance is added to the water. As depicted in Figure 1, other elements will compete for the available neutrons in a manner mathematically identical to parallel resistors competing for electric current.

The relevant quantity for our case is the multiple of an element's neutron capture cross section with its concentration in the water; this product is 
analogous to $\frac{1}{R}$. For example, chlorine has a neutron cross section of 33 barns, exactly 100 times that of hydrogen. Since there are two hydrogen atoms per water molecule, we would have to introduce one chlorine atom for every fifty water molecules in order to balance their capture rates (this is equivalent to setting $R_{1}=R_{2}$ in Figure 1 ).

On the plus side, chlorine gives off a very energetic gamma cascade with a total energy of $8.6 \mathrm{MeV}$ when it captures a neutron - easily enough to be seen in just about any water Cherenkov detector. However, if we used regular table salt, $\mathrm{NaCl}$, as a soluble source of our chlorine, then we would have to add three kilotons of salt to Super-Kamiokande's fifty kilotons of water in order to observe $50 \%$ of the neutron captures (the $50 \%$ which continue to capture on hydrogen are still invisible, of course). This is because the molecular weight of $\mathrm{NaCl}$ is more than three times that of $\mathrm{H}_{2} \mathrm{O}$, so one molecule of $\mathrm{NaCl}$ to every fifty water molecules means that about $6 \%$ of salt by weight is required. Such a quantity is just at the limit of practicality. Even worse, if we wanted $90 \%$ capture efficiency on chlorine instead of $50 \%$ we would need to put about 27 kilotons of salt in Super-K... clearly impossible.

Therefore, we have turned to the best neutron absorber known - gadolinium. Naturally occurring gadolinium (element number 64) has an absorption cross section of 49,700 barns, and has long been used in scintillator-based neutrino experiments near nuclear reactors. As far as we can determine, however, it has never before been proposed for use in a water Cherenkov detector. The reason for this, as well as the reasons why we believe it's now time to utilize it, will be discussed in the next two sections.

\section{Why Gadolinium Trichloride?}

\subsection{Solubility}

Metallic gadolinium is essentially insoluble in water and so is unacceptable in its pure form for use in a large water Cherenkov detector. However, the chloride compound of this rare earth, gadolinium trichloride or $\mathrm{GdCl}_{3}$ (sometimes known simply as gadolinium chloride), is readily soluble in water. 


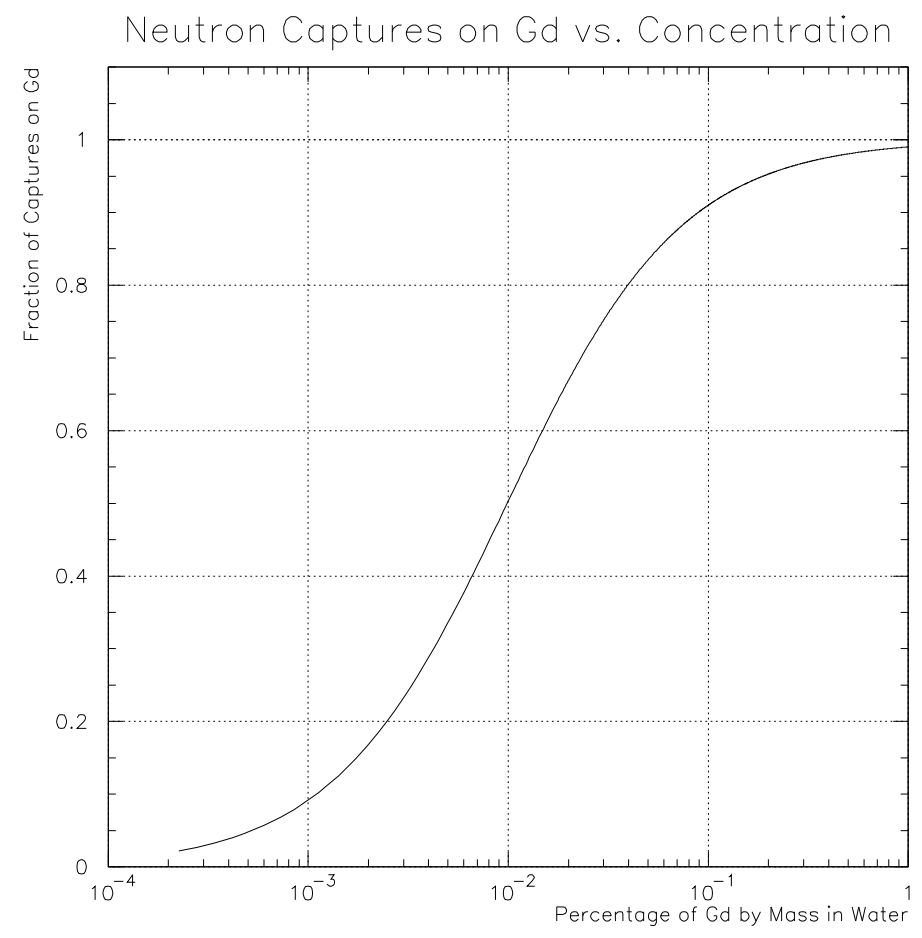

Figure 2: Fraction of neutrons visibly captured on gadolinium (instead of invisibly captured on hydrogen) versus the amount of $\mathrm{Gd}$ dissolved in light water.

\subsection{High Efficiency for Producing Observable $\gamma$-rays}

Gadolinium emits a healthy $8 \mathrm{MeV}$ gamma cascade when it captures a neutron. Like chlorine, this is enough energy to easily be seen in just about any large water Cherenkov detector. Unlike chlorine or $\mathrm{NaCl}$, however, the quantity of $\mathrm{GdCl}_{3}$ needed for high efficiency is not prohibitive. Working through the same calculation performed in Section 1.2 for $\mathrm{GdCl}_{3}$, we find that for 90\% neutron capture efficiency on gadolinium we need to mix only 90 tons of $\mathrm{GdCl}_{3}$ into the 50 kilotons of Super-K's water: less than $0.2 \%$ by weight. Figure 2 tells the tale.

Another nice thing about $\mathrm{GdCl}_{3}$ is that nothing goes to waste: the chlorine will contribute to the neutron capture efficiency, if only an additional $0.2 \%$ or so (we now have three "resistors" in parallel). Still, it's good to know that we will not introduce any elements into the ultra-pure water which will 
be merely isotopic bystanders. Furthermore, higher efficiencies than $90.2 \%$ are likely achievable via higher concentrations of $\mathrm{GdCl}_{3}$.

\section{Physics Reach of WC Detectors with $\mathrm{GdCl}_{3}$}

As attractive as $\mathrm{GdCl}_{3}$ looks from a neutron capture standpoint, there still must be some highly compelling physics reasons why we would want to modify a technology as mature and successful as that of large water Cherenkov detectors.

There are indeed quite a few highly compelling physics reasons why we would now like to be able to see neutrons in water Cherenkov detectors. They are the topic of this section.

\subsection{Relic Supernova Neutrinos}

Some 170,000 years ago, a blue supergiant in the Large Magellanic Cloud

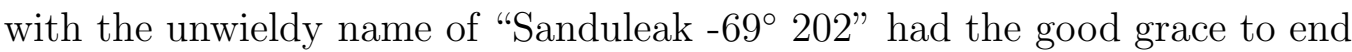
its life as a type II supernova. On February 23, 1987, the first radiation from the blast reached Earth. The blast, of course, became known as SN1987A, the first supernova observed anywhere near our galaxy since 1604 .

Three hours before the arrival of the light, a handful of neutrinos were seen via inverse beta decay, $\bar{\nu}_{e}+p \rightarrow e^{+}+n$ by one large water Cherenkov detector in Japan and one in the US. They saw only the Cherenkov light of nineteen emitted positrons: no information on the neutrons was collected.

While rather sparse, these first-of-their-kind observations absolutely energized the field of neutrino astrophysics. In fact, over the course of the following seventeen years there has appeared, on average, one publication every ten days based on these nineteen events. In 2002, Masatoshi Koshiba of the University of Tokyo was awarded the Nobel Prize in Physics for his role as the head of the Kamiokande experiment which first saw the neutrino burst from SN1987A.

Theorists and experimentalists alike wonder how we can get more data like this, as nearby supernovas are fairly rare events. However, all the neutrinos which have ever been emitted by every supernova since the onset of stellar formation suffuse the universe. These so-called "supernova relic neutrinos" [SRN], if observable, could provide a steady stream of information about not 
only stellar collapse and nucleosynthesis but also on the evolving size, speed, and nature of the universe itself.

Super-Kamiokande has recently conducted a search for these supernova relic neutrinos. However, this study was strongly background limited, especially by the many low energy events below $19 \mathrm{MeV}$ which swamped any possible SRN signal in that most likely energy range. Consequently, this study could see no statistically significant excess of events and therefore was only able to set the world's most stringent upper limits on the SRN flux.

If it were possible to look for coincident signals, i.e., for a positron's Cherenkov light followed shortly and in the same spot by the gamma cascade of a captured neutron, then these troublesome backgrounds could be almost completely eliminated. SRN models vary, but in principle Super-K with $\mathrm{GdCl}_{3}$ should then see a few of these events every year. A much larger, future detector like the proposed Hyper-Kamiokande would, with $\mathrm{GdCl}_{3}$ in its water, collect an amount of SRN events equal to what was seen seventeen years ago from SN1987A every month or so.

\subsection{Galactic Supernova Neutrinos}

If we are fortunate enough to observe a nearby supernova in the coming decades, it would be most beneficial to have $\mathrm{GdCl}_{3}$ in the water of the large water Cherenkov detectors which are online when the resulting neutrino wave sweeps across the planet. This is because the most copious supernova neutrino signal by far $(\sim 80 \%)$ comes from inverse beta events. They are only produced by one of the six species of neutrinos and anti-neutrinos which are generated by a stellar collapse, and so if we could tag them individually by their follow-on neutron captures then we could extract the $\bar{\nu}_{e}$ time structure of the burst precisely. What's more, we could then subtract them away from the more subtle non- $\bar{\nu}_{e}$ signals, uncovering additional information that would otherwise be lost from this once-in-a-lifetime happening.

\subsection{Reactor Anti-neutrinos}

Nuclear power reactors produce prodigious quantities of low energy (below $8 \mathrm{MeV}$ ) anti-neutrinos. The neutrino was first discovered by using a reactor as a source, and in the years which followed the properties of $\bar{\nu}_{e}$ 's have been examined with increasing accuracy and precision by a series of scintillatorbased short-baseline reactor experiments. Finally, in what appeared to many 
to be the ultimate reactor neutrino experiment, the KamLAND experiment began operations a few years ago and has already provided strong evidence that the Large Mixing Angle MSW neutrino oscillation solution is the correct one to explain the long-standing Solar Neutrino Problem.

But KamLAND may not be the last word in reactor $\bar{\nu}_{e}$ 's, after all. If we were to introduce a $0.2 \%$ solution of $\mathrm{GdCl}_{3}$ into Super-Kamiokande, we could collect enough reactor anti-neutrino data to reproduce their first published results in just three days of operation. Their entire planned six-year datataking run could be reproduced by Super-K with $\mathrm{GdCl}_{3}$ in seven weeks.

This should, especially given the extremely high statistics involved, allow significantly tighter constraints to be placed on the solar neutrino oscillation parameters than any other method which could conceivably become operational before the close of the present decade, and possibly far beyond.

Figure 3 shows the expected spectrum of neutron-tagged positrons in a $\mathrm{GdCl}_{3}$-enriched Super-K. The width of the band labeled "Supernovae" reflects the remaining allowed range of theoretical flux predictions for the as-yet-unobserved relic supernova $\bar{\nu}_{e}$ 's.

\subsection{Atmospheric Neutrinos}

In addition to the observation of supernova neutrinos from SN1987A, large water Cherenkov detectors are probably best known for their Standard Modelshaking discovery of atmospheric neutrino oscillations.. One problem they suffer from, however, is a difficulty in differentiating between atmospheric neutrinos and atmospheric anti-neutrinos. This nagging flaw has led to some grandiose and likely unworkable schemes involving the placement of huge, powerful magnetic fields next to and/or within the next generation of experiments. As existing photomultiplier tubes will not operate in the presence of even the Earth's own weak field (Super-K's entire volume is carefully compensated via massive high-current coils which surround the detector and exactly cancel out the Earth's field) this method of matter/anti-matter identification appears quite impossible for water Cherenkov detectors.

With neutron-detecting $\mathrm{GdCl}_{3}$ in the water, however, the reactions $\bar{\nu}_{e}+$ $H \rightarrow e^{+}+n, \bar{\nu}_{\mu}+H \rightarrow \mu^{+}+n$, and $\bar{\nu}_{\tau}+H \rightarrow \tau^{+}+n$ are clearly distinct from the reactions $\nu_{e}+n \rightarrow e^{-}+p, \nu_{\mu}+n \rightarrow \mu^{-}+p$, and $\nu_{\tau}+n \rightarrow \tau^{-}+p$, where the resulting proton generally produces no light. By collecting a sample of neutron-tagged atmospheric anti-neutrino events we would be able to directly compare their flux and zenith angle dependence with that of the usual, mixed 


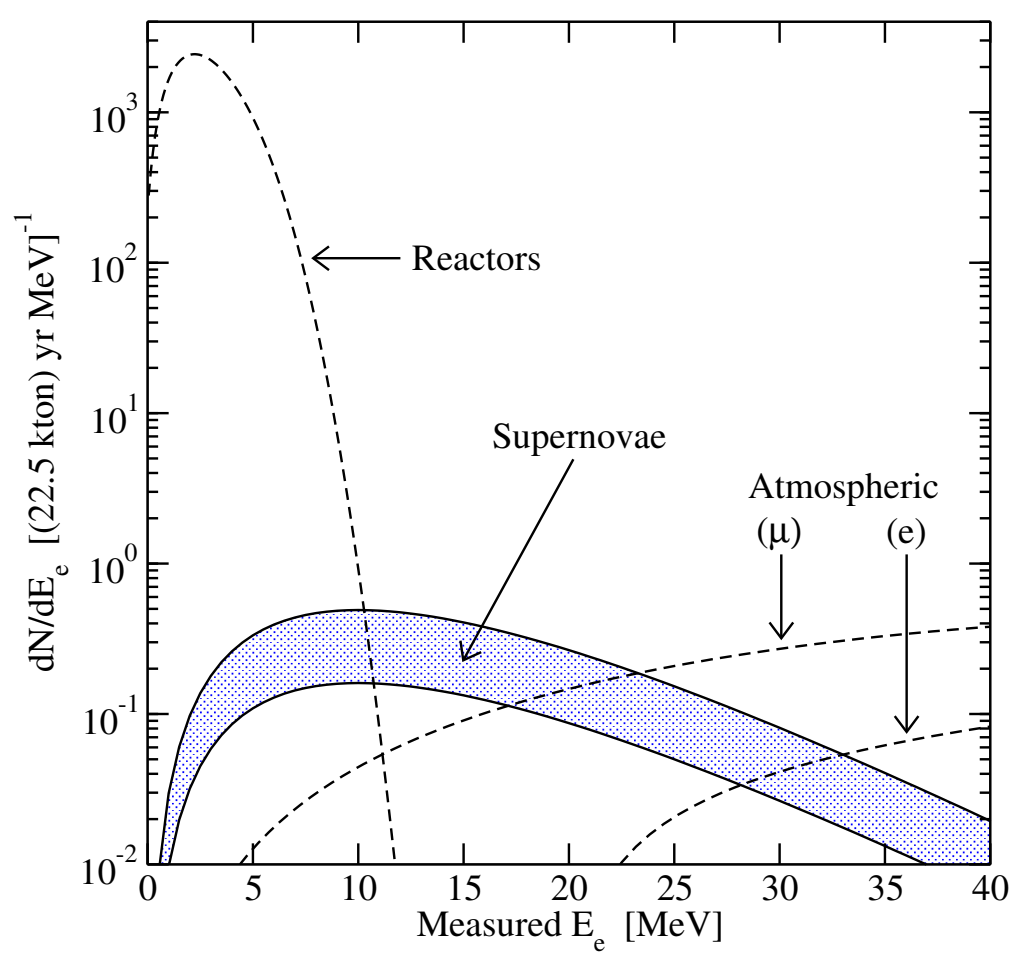

Figure 3: Expected positron spectrum tagged by neutron captures in a $\mathrm{GdCl}_{3}$ -enriched Super-Kamiokande. One year of data is shown, with SK's energy resolution and all known backgrounds taken into account. Note the huge reactor antineutrino signal, and the clear window for observing the relic supernova neutrinos between the reactor events and the tail end of the neutron-liberating atmospheric neutrino events.

neutrino sample, thereby testing whether or not neutrinos and anti-neutrinos are behaving in the same way.

\subsection{Long Baseline Neutrinos}

Similar to the case of the atmospheric neutrinos, the ability to differentiate between neutrinos and anti-neutrinos would clearly benefit tests of oscillations and future measurements of the elusive neutrino mixing angle $\theta_{13}$ and what is perhaps the ultimate challenge of neutrino physics, the eventual measurement of the neutrino CP phase parameter. 


\subsection{Proton Decay}

Large water Cherenkov detectors got their start as proton decay experiments (in Kamiokande the last three letters stood for "nucleon decay experiment," while in Super-Kamiokande the same letters stand for "neutrino detection experiment") and still provide by far the most sensitive measurements of the proton and bound neutron lifetimes. The next generation of detectors such as Hyper-Kamiokande and UNO are designed to push these limits even farther, hopefully observing a positive nucleon decay signal at last.

In order to positively identify such a vanishingly rare process, however, these detectors will have to do everything possible to keep their backgrounds in check. These backgrounds are almost entirely due to atmospheric neutrino interactions which can occasionally mimic a true nucleon decay signal. As the atmospheric neutrinos cannot be shielded, one must live with them and their interactions. While more detailed study is needed, we believe that gadolinium's ability to spot free neutrons, neutrons which should almost never be produced by true nucleon decay events, will provide a useful new background reduction technique for these physics frontier projects.

\section{Program of Study}

In the previous section we discussed the many powerful physics advantages gained by adding $\mathrm{GdCl}_{3}$ to large water Cherenkov detectors. However, there are some implementation issues which need to be investigated before this new physics can be realized in Super-Kamiokande. In order to help study them, we proposed a large-scale test including the following steps:

1. Employ the knowledge gained over the previous two years of DOEfunded bench tests at UCI regarding $\mathrm{GdCl}_{3}$ filtration to modify the one kiloton water Cherenkov detector which served as a near detector for the $\mathrm{K} 2 \mathrm{~K}$ long-baseline neutrino oscillation experiment in Japan. These modifications would enable the water filtration system of the one kiloton detector to hold $\mathrm{GdCl}_{3}$ concentrations in the water fixed while cleaning out all other impurities.

2. Measure the effect of $\mathrm{GdCl}_{3}$ on UV light propagation in water as a function of concentration by utilizing the one kiloton tank's 680 20inch photomultiplier tubes and calibration laser injectors. 


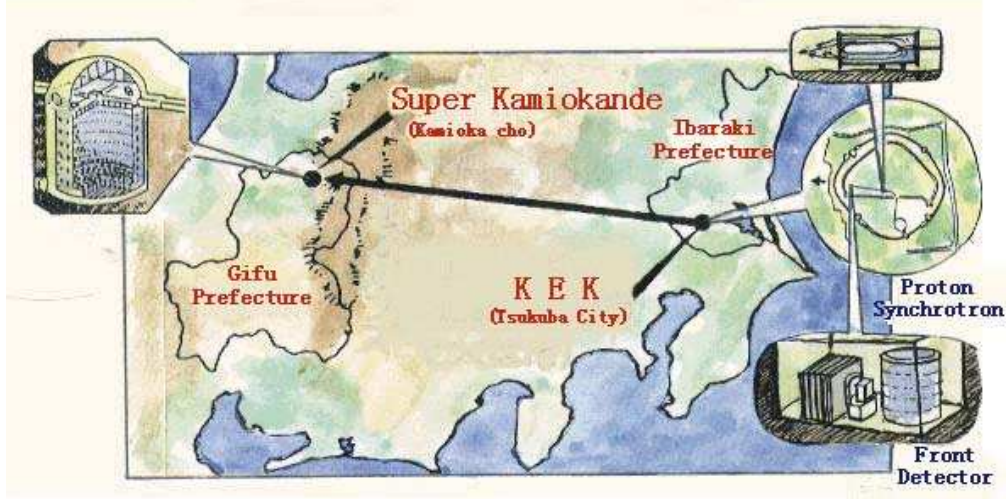

Figure 4: Overview of the K2K Long-baseline neutrino oscillation experiment. The one kiloton detector is the cylindrical object in the front detector.

3. Study the effect of $\mathrm{GdCl}_{3}$ exposure on PMT glass, cabling, and other materials in the one kiloton tank. These are the same materials found in Super-Kamiokande and other water Cherenkov detectors.

\subsection{The One Kiloton Detector at KEK}

A major piece of surplus equipment became available in 2005 - the one kiloton tank from the K2K long-baseline neutrino oscillation experiment. Figure 4 shows an overview of this project. It first took data in 1999 and, having confirmed Super-K's 1998 observation of atmospheric neutrino oscillations, permanently ceased operations in early 2005.

As seen in Figure 5, this one kiloton tank was essentially a $2 \%$ scale model of Super-Kamiokande itself. It was designed to reduce systematic errors as much as possible when comparing the unoscillated neutrino signal at the end of the beamline's neutrino production volume to the (presumably) oscillated signal received at Super-K some 250 kilometers distant. As such, this smaller detector used the same electronics, photomultiplier tubes, etc., as its 50 kiloton downstream cousin. Figure 6 is an interior view of the detector.

The PMT's, power supplies, DAQ electronics, and online computing facilities for the one kiloton detector were owned by the University of Tokyo's Institute for Cosmic Ray Research [ICRR], the same organization which owns and operates Super-Kamiokande. 


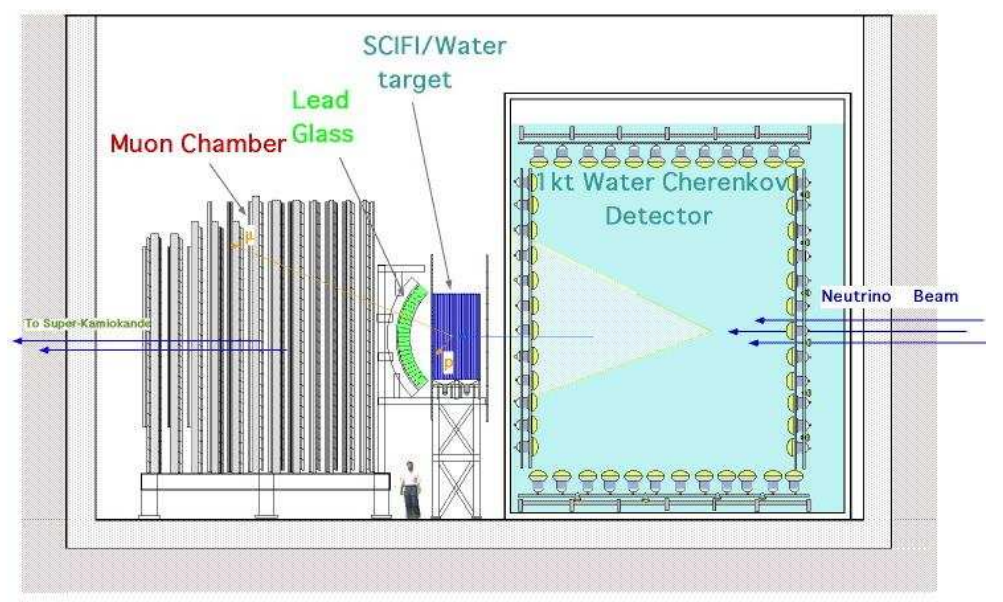

Figure 5: The front detectors of the $\mathrm{K} 2 \mathrm{~K}$ experiment. They are located in an underground hall at the end of the neutrino production region.

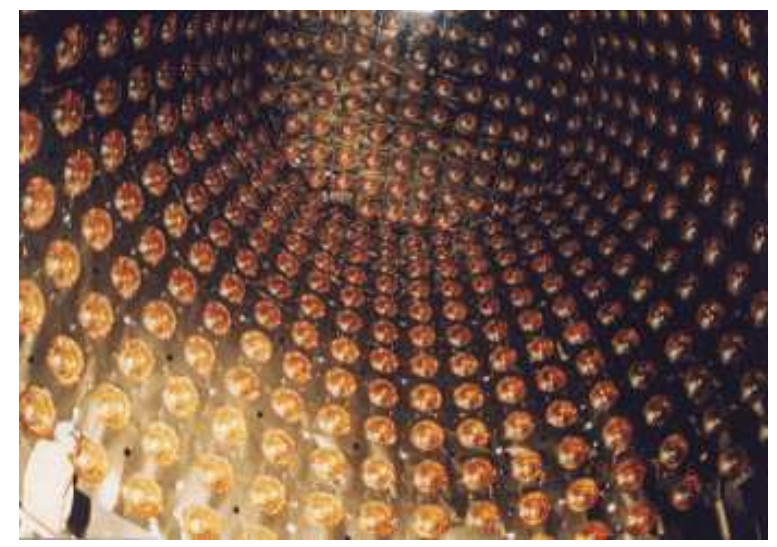

Figure 6: Interior of K2K's one kiloton water Cherenkov detector. Many of the 680 20-inch Hamamatsu photomultipliers, the same type as those used in Super-Kamiokande, are easily seem.

The one kiloton [1KT] detector had another attractive feature - its water filtration system was originally built from the ground up and continued to be maintained by UCI. This made the needed $\mathrm{GdCl}_{3}$-related modifications relatively easy for us.

We were awarded a 2005 Advanced Detector Research Program grant (DOE Grant Number: DE-FG02-05ER41386) for the large-scale study of 
these three topics. What follows are the results of our 2005-2006 R\&D program.

\section{Results of the Study}

\subsection{Water Transparency in the $1 \mathrm{KT}$}

In November of 2005 we added 200 kilograms of $\mathrm{GdCl}_{3}$ to the kiloton detector at $\mathrm{KEK}$. Injecting the dissolved $\mathrm{GdCl}_{3}$ into the bottom of the tank took just a few minutes, but given the recirculation rate of 22 tons/hour it took two days to fully mix the injected $\mathrm{GdCl}_{3}$ solution with the rest of the water.

This yielded an overall $\mathrm{GdCl}_{3}$ concentration of $0.02 \%$ - one tenth the eventual planned $0.2 \%$ concentration in Super-K, but still capable of capturing $50 \%$ of the ambient neutrons on gadolinium (see Figure 2). Assuming no problems arose we were ready to add (and had available at KEK) another $1,800 \mathrm{~kg}$ of $\mathrm{GdCl}_{3}$ in order to bring its concentration up to the full design level of $0.2 \%$. However, problems having little to do with the study at hand did arise.

Although pre-existing rust was eventually lifted into solution and made further measurements impossible, we saw a drop in collected charge (which is directly related to the amount of light reaching the phototubes) of less than $1.5 \%$ between the time when the detector was full of pure water and the time when the $\mathrm{GdCl}_{3}$ was uniformly distributed within it. Some of this loss was undoubtedly due to the action of the pre-existing rust, however. Figure 7 shows the total collected charge for vertical throughgoing cosmic ray muons in the kiloton detector just before and just after the addition of $200 \mathrm{~kg}$ of $\mathrm{GdCl}_{3}$ in November of 2005. Overlaid is the concentration of chlorine from the $\mathrm{GdCl}_{3}$ in the return water taken from the top of the tank. Dissolved gadolinium levels, though not shown in the figure, were also measured and track in an identical way as the chlorine.

Note that the point at which the collected charge begins to drop rapidly, the result of a large band of pre-existing rust at the top of the kiloton tank coming into contact with the $\mathrm{GdCl}_{3}$ and then going into solution, occurred immediately after the $\mathrm{GdCl}_{3}$ was fully mixed within the entire volume of the detector as evidenced by the chlorine output level becoming steady. Therefore, we may conclude that the $\mathrm{GdCl}_{3}$ itself did not degrade the transparency of the water by more than the initial $1.5 \%$ drop seen during the two days 


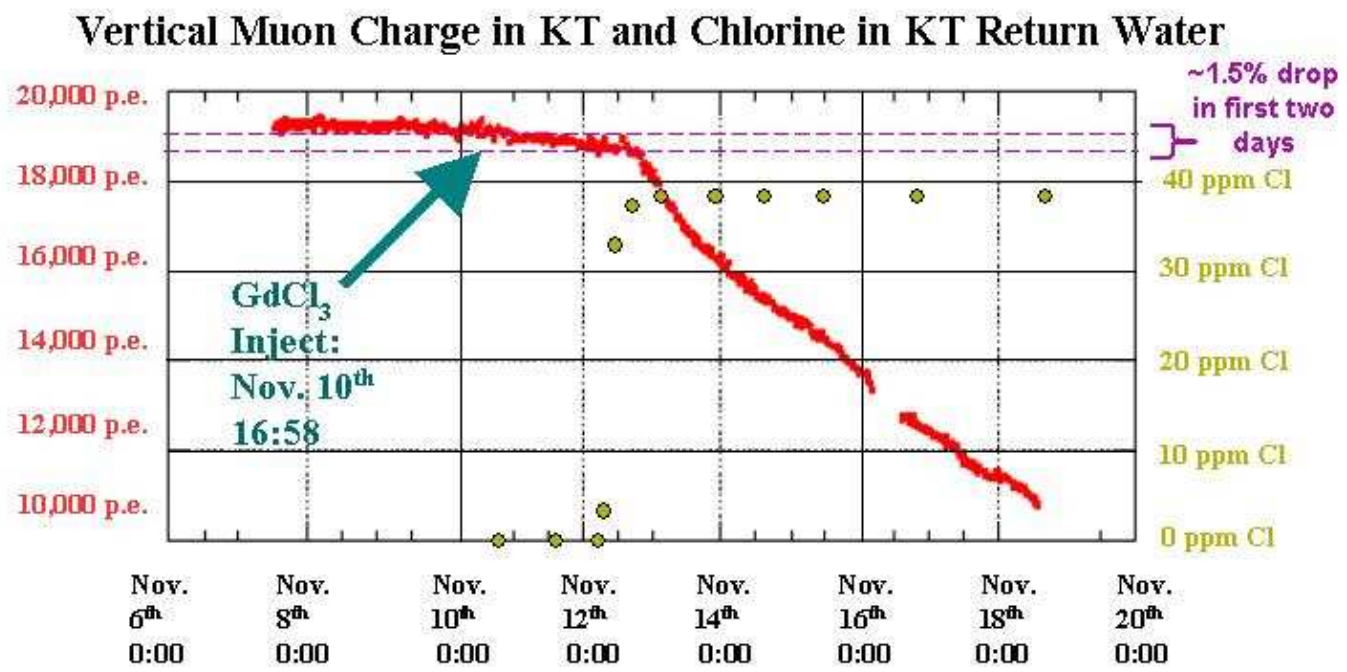

Figure 7: This plot shows the average total collected charge for throughgoing muons in the kiloton detector just before and just after the addition of $200 \mathrm{~kg}$ of $\mathrm{GdCl}_{3}$. Overlaid is the concentration of chlorine from the $\mathrm{GdCl}_{3}$ in the return water. There is a $1.5 \%$ reduction in total light seen while the $\mathrm{GdCl}_{3}$ was being fully mixed within the entire volume of the detector.

required to evenly distribute it throughout the tank's volume.

Unfortunately, seven years of exposure to ultra-pure water during the entire $\mathrm{K} 2 \mathrm{~K}$ experimental run ultimately rendered the $1 \mathrm{KT}$ unsuitable for precision $\mathrm{GdCl}_{3}$ studies. In late March of 2006 the $\mathrm{GdCl}_{3}$ was removed from solution (see Figures 8 and 9), the tank was drained for the first time since 1998, and an inspection was performed. As can be seen in Figure 10, we found that large areas (about 20\% of the total inner surface area) had not been properly painted back in 1998, leaving the iron surface exposed and, by 2005, covered with a thick layer of rust. This was rapidly lifted into solution by the $\mathrm{GdCl}_{3}$, and caused complicating effects.

Note that the $\mathrm{GdCl}_{3}$ itself did not cause the rust. This has been checked with tabletop tests involving clean and pre-rusted iron samples soaked in $\mathrm{GdCl}_{3}$ solutions. As Super-K is made of high-grade stainless steel, not 


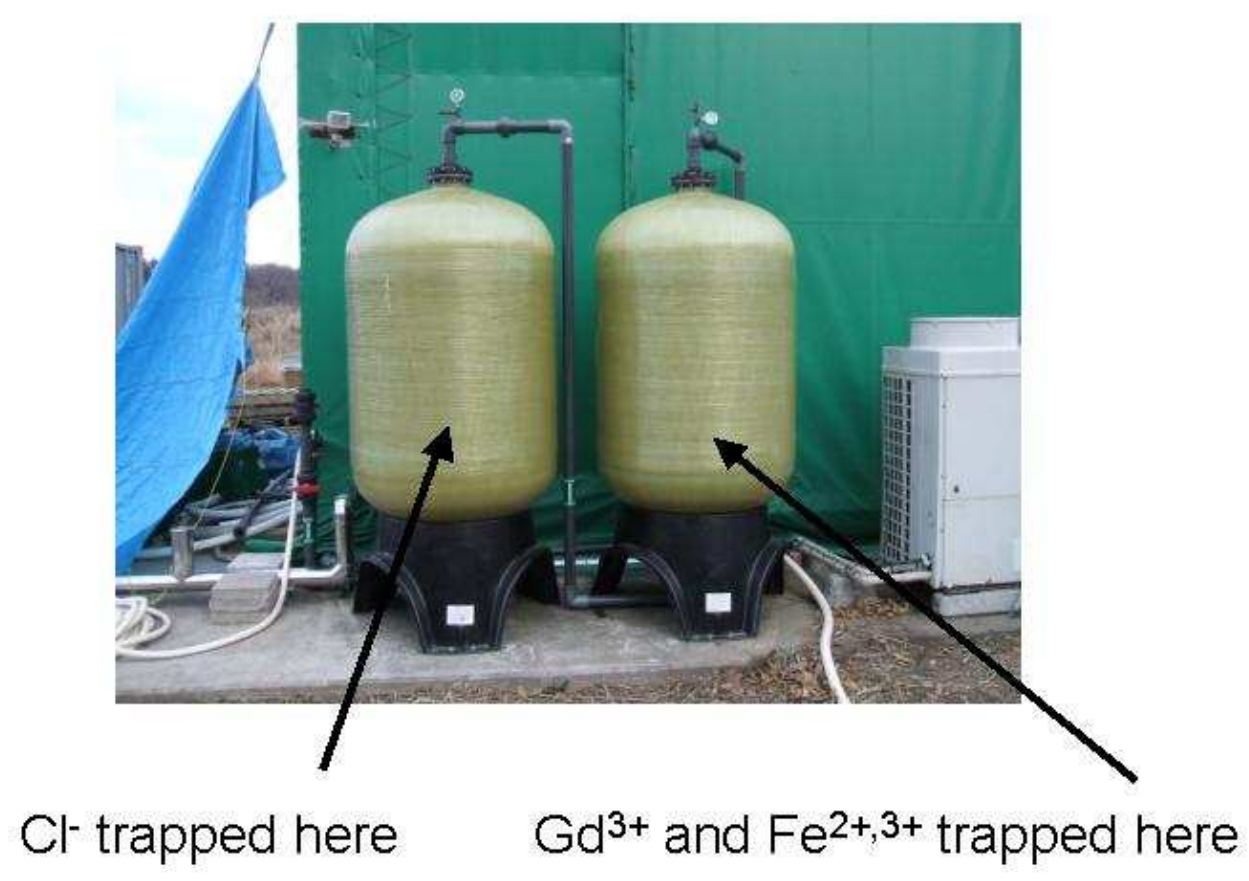

Figure 8: Anion and cation ion exchange resin tanks used to remove $\mathrm{GdCl}_{3}$ from the $1 \mathrm{KT}$. A single pass through this system removed $>99 \%$ of all ions, producing nine megaohm water - essentially the same as that in the SK tank. Within two weeks this system had removed all $\mathrm{GdCl}_{3}$ from the $1 \mathrm{KT}$.

(badly) painted iron, we expect no such problems there. A corrosion report by Mitsui Heavy Industries, the Japanese shipbuilding company who built the Super-K tank, also supports this conclusion. As a result, gadoliniuminduced corrosion is now expected to be insignificant in SK. The pristine condition of the $1 \mathrm{KT}$ photomultiplier tubes and their stainless steel support structure following four months of exposure to $\mathrm{GdCl}_{3}$ (see Figure 11) supports this conclusion as well.

\subsection{Water Purification System of the $1 \mathrm{KT}$}

Based on our studies at UCI and KEK, we developed a method using reverse osmosis [RO] by which water with dissolved $\mathrm{GdCl}_{3}$ may be split into two streams - one containing essentially no $\mathrm{GdCl}_{3}$ and one containing highly 


\section{Vertical Muon Charge in KT and Chlorine in KT Return Water}

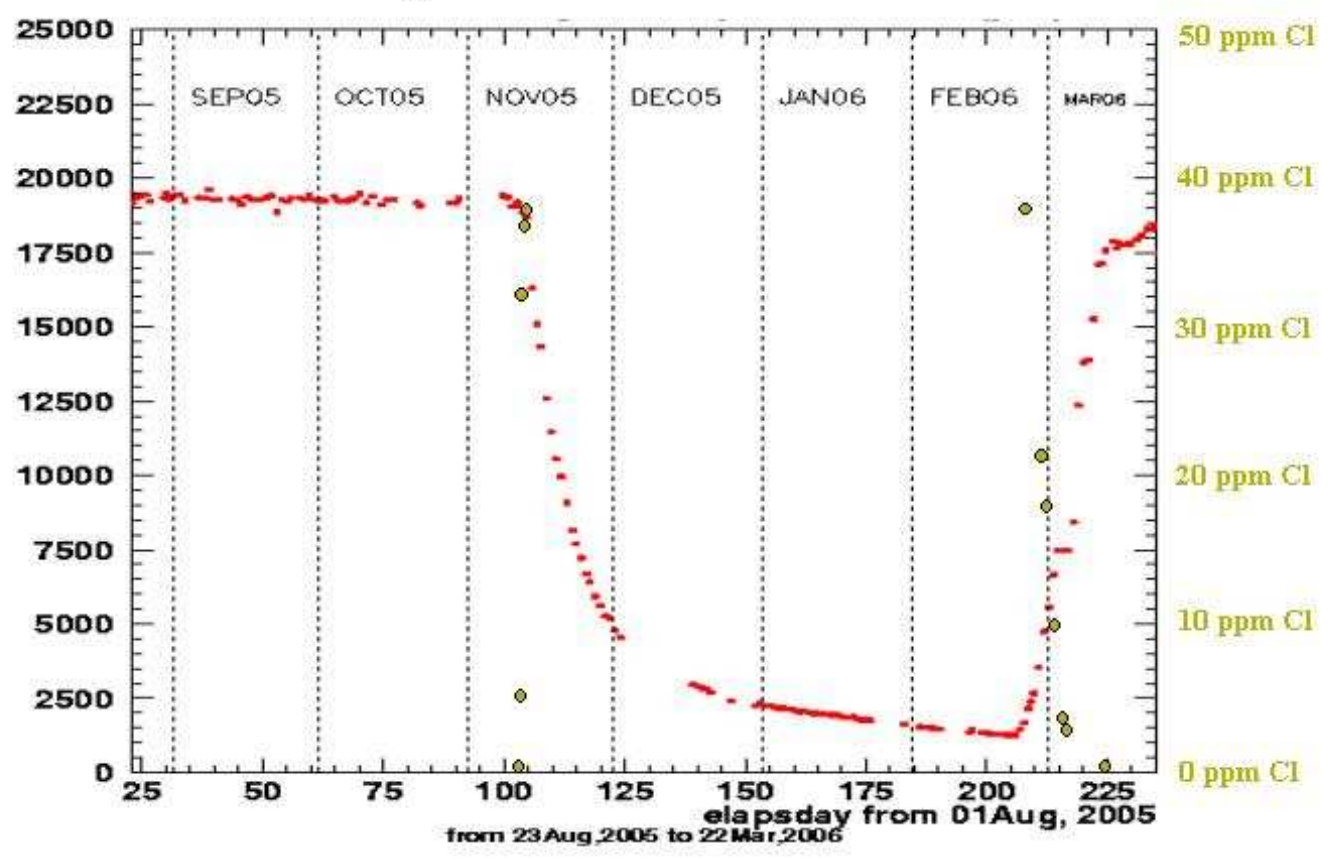

Figure 9: The average total collected charge for throughgoing muons in the kiloton detector during the entire period of the $\mathrm{GdCl}_{3}$ test is shown. Overlaid is the concentration of chlorine from the $\mathrm{GdCl}_{3}$ in the return water. Note that the collected light improved back towards its pre- $\mathrm{GdCl}_{3}$ level quite rapidly upon removal of the $\mathrm{GdCl}_{3}$ and the dissolved iron. The water quality was still improving at the point at which the tank was drained for inspection on March 22nd, 2006.

concentrated $\mathrm{GdCl}_{3}$. The philosophy behind this work was that some components of the existing SK water system like the vacuum degassifier will not operate correctly when exposed to significant amounts of dissolved materials, while other components like the deionization mix beds will remove the $\mathrm{GdCl}_{3}$ from the water altogether. With a split stream, the "Gd-safe" water system components can be fed the water containing $\mathrm{GdCl}_{3}$, while the "Gd-unsafe" components can be fed the water without $\mathrm{GdCl}_{3}$. In this way the SK water will continue to be purified, but the $\mathrm{GdCl}_{3}$ will remain in solution.

The equipment developed at UCI was installed in the water system at the 


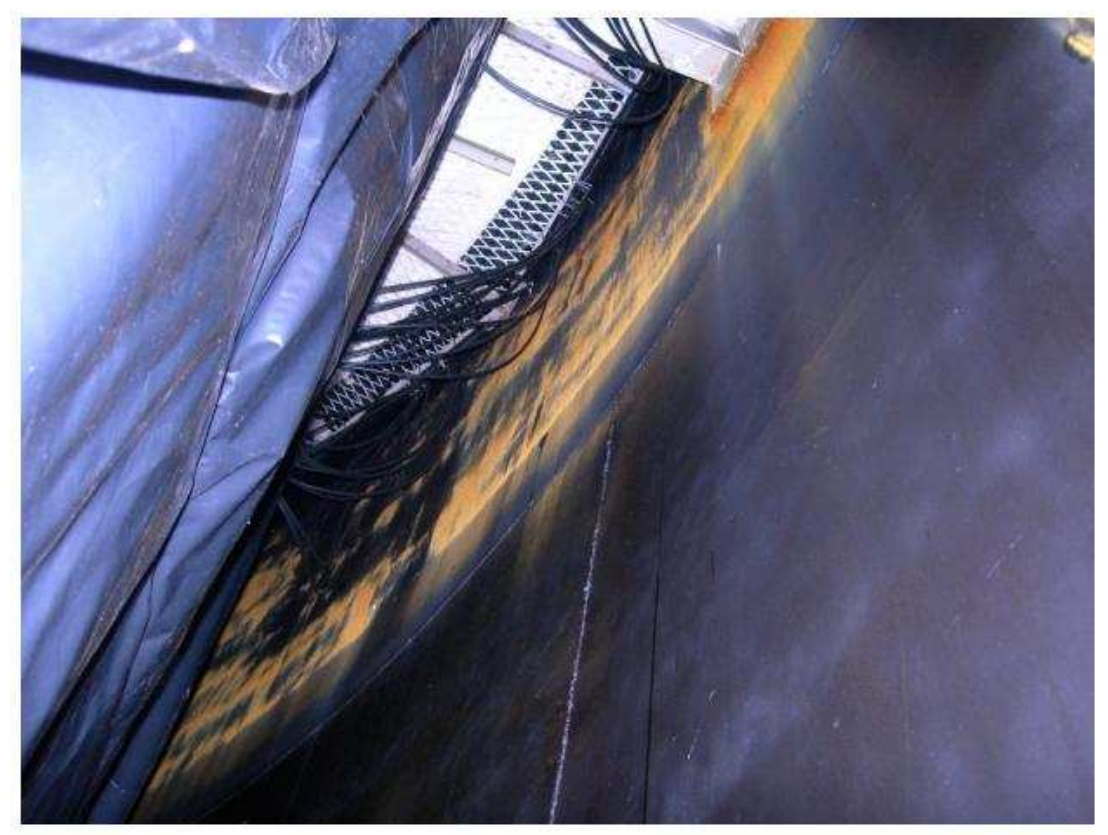

Figure 10: Picture of the inner wall of the kiloton tank showing a 1.5 meter tall band of rust near the top of the detector. The iron surface in this region had not been properly painted in 1998, allowing rust to form over years of exposure to ultra-pure water.

KT and tested there. Figure 12 shows a schematic of the actual water system implementation used at KEK. It performed perfectly, removing $>99.9 \%$ of the $\mathrm{GdCl}_{3}$ at a flow rate of over 3.5 tons/hour. A similar modification to the SK system would mostly involve the addition of more plumbing to the existing hardware, as a large RO unit is already in place there.

\subsection{Conclusions}

It was most unfortunate that the $1 \mathrm{KT}$ proved to be an inappropriate device in which to study the properties of $\mathrm{GdCl}_{3}$. Had it not been for the improperly treated inner surface of the tank we would have been able to observe the behavior of a large, Gd-enriched water Cherenkov detector, and had all gone well we would likely now stand ready, at least from a technical stand- 


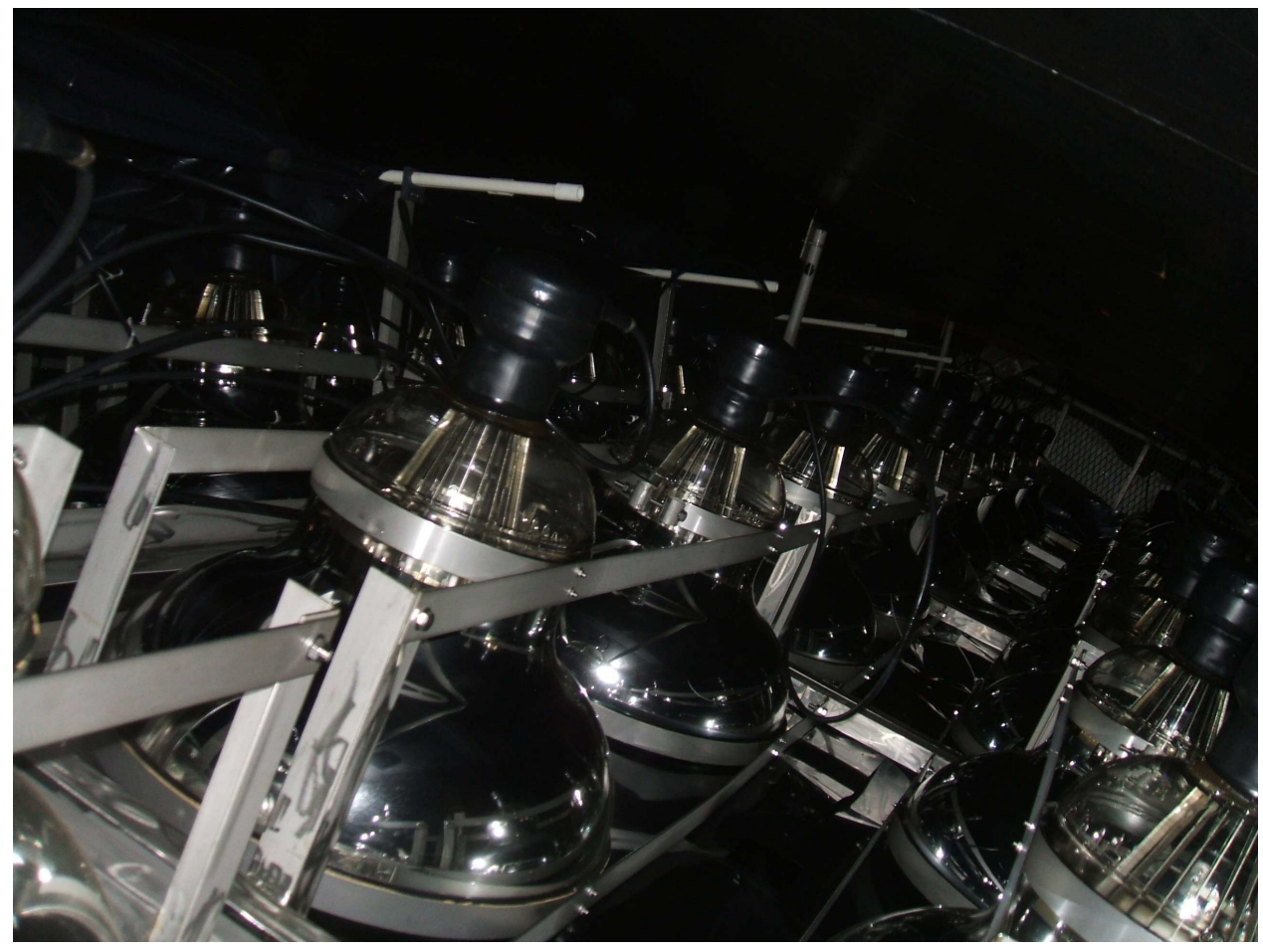

Figure 11: Picture of the one kiloton detector's wall of PMT's (as seen from behind) after a four month exposure to $\mathrm{GdCl}_{3}$. Note the shiny appearance and complete lack of rust or other damage to the stainless steel support structure.

point, to introduce $\mathrm{GdCl}_{3}$ into Super-K. Nevertheless, we learned a number of important things from putting $200 \mathrm{~kg}$ of $\mathrm{GdCl}_{3}$ into the kiloton detector in 2005:

- $\mathrm{GdCl}_{3}$ is extremely easy to dissolve in water. With vigorous stirring, $200 \mathrm{~kg}$ was dissolved in 2,000 liters of water (yielding a concentrated $10 \%$ solution of $\mathrm{GdCl}_{3}$ ) in around 10 minutes.

- The $\mathrm{GdCl}_{3}$ itself (i.e., in the absence of old rust) does not drastically affect the light collection. As discussed above, an initial $\sim 1.5 \%$ drop in total collected light was seen after the $\mathrm{GdCl}_{3}$ was added, but what fraction of that was due to the $\mathrm{GdCl}_{3}$ and what was due to the lifted rust was not clear.

- Choice of detector materials is critical with $\mathrm{GdCl}_{3}$. 


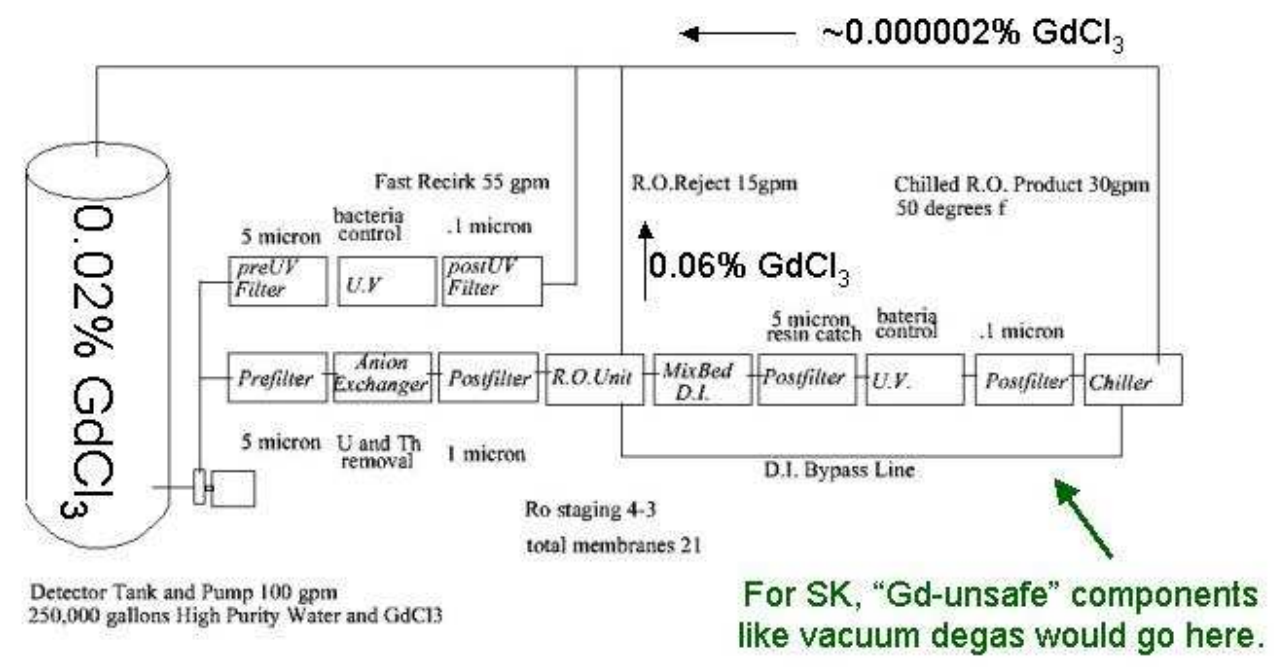

Figure 12: Schematic of the water purification system, suitable modified to handle dissolved $\mathrm{GdCl}_{3}$, which was used during the KT test at KEK. The system separated the $\mathrm{GdCl}_{3}$-enrich and the pure water into two streams via the use of reverse osmosis. It separated over 3.5 tons/hour of dissolved $\mathrm{GdCl}_{3}$ without any trouble, even in the presence of dissolved iron.

- The 20-inch Super-K PMT's operate well in conductive water. During four months of exposure to the $\mathrm{GdCl}_{3}$ none of these PMT's (out of 680) failed, and they produced the same amount of charge at the end of the test once the water became clear again as they did at the beginning (see Figure 9).

- Our RO-based Gd filtration system works as designed at 3.6 tons/hr (the remaining 18 tons/hr was passed through a fast-recirculation circuit for cooling and UV treatment purposes) and can easily be scaled up to higher (Super-K level) flows. 